\title{
INDEX TO VOLUME 77
}

\section{RESEARCH ANNOUNCEMENTS}

Abrahamse, M. B. Toeplitz operators in multiply connected regions, 449.

Adams, D. R. and Meyers, N. G. Bessel potentials. Inclusion relations among classes of exceptional sets, 968.

Agoston, M. K. The reducibility of Thom complexes and surgery on maps of degree $d, 106$.

Akiba, Tadatoshi. Homotopy types of some PL complexes, 1060.

Allard, W. K. A regularity theorem for the first variation of the area integrand, 772 .

Anshel, Michael. The endomorphisms of certain one-relator groups and the generalized Hopfian problem, 348.

Antonelli, P., Burghelea, D. and Kahn, P. J. Concordance-homotopy groups and the nonfinite type of some Diff $M^{n}, 719$.

Argabright, Loren and Gil de Lamadrid, Jesús. Fourier transforms of unbounded measures, 355 .

Ash, J. M. and Welland, G. V. Convergence, summability, and uniqueness of multiple trigonometric series, 123.

Ault, Janet and Petrich, Mario. The structure of w-regular semigroups, 196.

Baker, K. A. Equational axioms for classes of lattices, 97.

Barratt, M. G. On F-structures, 1099.

Berenstein, C. A. and Dostal, M. A. Fourier transforms of the Beurling classes $D_{\omega}$, $E_{\omega}, 963$.

Berman, Stephen. Abstract wave equations with finite velocity of propagation, 1001.

Billotti, J. E. and LaSalle, J. P. Dissipative periodic process, 1082.

Bousfield, A. K. and Kan, D. M. Localization and completion in homotopy theory, 1006.

Brezis, H., Rosenkrantz, W. and Singer, B. An extension of Khintchine's estimate for large deviations to a class of Markov chains converging to a singular diffusion, 980.

Brillhart, John. See Morrison, M. A.

Brooks, J. K. On the existence of a control measure for strongly bounded vector measures, 999.

Browder, F. E. Normal solvability for nonlinear mappings into Banach spaces, 73.

Browder, W. and Brumfiel, G. A note on cobordism of Poincaré duality spaces, 400.

Browder, William and Petrie, Ted. Diffeomorphisms of manifolds and semifree actions on homotopy spheres, 160.

Browder, William, Petrie, Ted and Wall, C. T. C. The classification of free actions of cyclic groups of odd order on homotopy spheres, 455 .

Brumfiel, G. See Browder, W.

Brumfiel, G., Madsen, I. and Milgram, R. J. PL characteristic classes and cobordism, 1025.

Burghelea, D. See Antonelli, P.

Cappell, Sylvain. A splitting theorem for manifolds and surgery groups, 281.

Chartrand, Gary and Schuster, Seymour. On the existence of specified cycles in complementary graphs, 995.

Chase, S. U. On inseparable Galois theory, 413.

Chen, B.-Y. On an inequality of mean curvatures of higher degree, 157.

On the mean curvature of submanifolds of euclidean space, 741.

Chillingworth, D. R. J. and Hubbard, J. A note on nonrigid Nash structures, 429.

Chow, Kwang-nan. Minimality in families of solutions of $\Delta u=P u$ on Riemannian manifolds, 1079. 
Chow, S.-N. and Schuur, J. D. An existence theorem for ordinary differential equations in Banach spaces, 1018.

Clough, Robert and Stasheff, James. BSJ does not map correctly into BSF mod 2, 1072.

Colton, David. Bergman operators for elliptic equations in three independent variables, 752.

Czerniakiewicz, Anastasia. Automorphisms of a free associative algebra of rank 2, 992.

Delver, Robert. Variational problems within the class of solutions of a partial differential equation, 1008.

Diestel, Joseph, Morris, S. A. and Saxon, S. A. Varieties of locally convex topological vector spaces, 799.

Dostal, M. A. See Berenstein, C. A.

Dubuc, E. J. and Porta, Horacio. Convenient categories of topological algebras, 975.

Dudley, R. M. On measurability over product spaces, 271.

Dunkl, C. F. and Ramirez, D. E. $C^{*}$-algebras generated by measures, 411.

Dwyer, T. A. W., III. Partial differential equations in Fischer-Fock spaces for the HilbertSchmidt holomorphy type, 725.

Ehrenfeucht, Andrzej and Mycielski, Jan. Abbreviating proofs by adding new axioms, 366.

Eklund, N. A. Boundary behavior of solutions of parabolic equations with discontinuous coefficients, 788.

Eliasson, H. I. Condition (C) and geodesics on Sobolev manifolds, 1002.

Elman, Richard and Lam, T. Y. Pfister forms and K-theory of fields, 971.

Erickson, K. B. A renewal theorem for distributions on $R^{1}$ without expectation, 406.

Faith, Carl. A correspondence theorem for projective modules and the structure of simple noetherian rings, 338.

- Orders in semilocal rings, 960.

Farias, A. O. Orientation-preserving mappings, a semigroup of geometric transformations and a class of integral operators, 422.

Fefferman, Charles. On the divergence of multiple Fourier series, 191.

- Characterizations of bounded mean oscillation, 587.

- On the convergence of multiple Fourier series, 744.

Fields, K. L. On the Brauer-Speiser theorem, 223.

- On the Schur subgroup, 477.

Flanders, Harley. Infinite resistive networks, 564.

Fleischner, Herbert. The square of every nonseparable graph is Hamiltonian, 1052.

Fogarty, John. Fixed point schemes, 203.

Gamelin, T. W. and Garnett, John. Pointwise bounded approximation and hypodirichlet algebras, 137.

Garnett, John. See Gamelin, T. W.

Geoghegan, Ross. Manifolds of piecewise linear maps and a related normed linear space, 629.

Gersten, S. M. Homotopy theory of rings and algebraic K-theory, 117.

Gersten, S. M. and Rector, D. L. A relation between two simplicial algebraic $K$-theories, 397.

Gil de Lamadrid, Jesús. See Argabright, Loren.

Glasner, Moses, Katz, Richard and Nakai, Mitsuru. A remark on classification of Riemannian manifolds with respect to $\Delta u=P u, 425$.

Gluck, Herman. Deformations of normal vector fields and the generalized Minkowski problem, 1106. 
Golubitsky, Martin and Rothschild, Bruce. Primitive subalgebras of exceptional Lie algebras, 983.

Gordon, C. McA. A short proof of a theorem of plans on the homology of the branched cyclic coverings of a knot, 85 .

Graef, J. R. Boundedness and oscillation of solutions of the Liennard equation, 418.

Graves, William. An algebra associated to a combinatorial geometry, 757.

Greene, R. E. and Wu, H. Curvature and complex analysis, 1045.

Greenfield, S. J. and Wallach, N. R. The Hilbert ball and bi-ball are holomorphically inequivalent, 261.

Grimm, L. J. Existence and uniqueness for nonlinear neutral-differential equations, 374.

Gromoll, Detlef and Wolf, J. A. Some relations between the metric structure and the algebraic structure of the fundamental group in manifolds of nonpositive curvature, 545.

Gross, Leonard. A noncommutative extension of the Perron-Frobenius theorem, 343.

Guggenheimer, H. Geometric theory of differential equations. The Ljapunov integral for monotone coefficients, 765.

Gulliver, Robert, II. On regularity of generalized surfaces of constant mean curvature, 243.

Hagopian, C. L. A fixed point theorem for plane continua, 351.

-. Semiaposyndetic nonseparating plane continua are arcwise connected, 593.

Harvey, F. R. and Wells, R. O., Jr. Holomorphic approximation on totally real submanifolds of a complex manifold, 824.

Hejhal, D. A. Classification theory for Hardy classes of analytic functions, 767.

Hess, Peter. Nonlinear functional equations in Banach spaces and homotopy arguments, 211.

Howe, Roger. Representation theory for division algebras over local fields (tamely ramified case), 1063.

Hsiang, Wu-yi. Some fundamental theorems in cohomology theory of topological transformation groups, 1094.

Hubbard, J. See Chillingworth, D. R. J.

Hughes, C. E., Oberbeek, Ross and Singletary, W. E. The many-one equivalence of some general combinatorial decision problems, 467.

Jech, T. J. and Powell, W. C. Standard models of set theory with predication, 808.

Kahn, P. J. See Antonelli, P.

Kan, D. M. See Bousfield, A. K.

Katz, Richard. See Glasner, Moses.

Kawakubo, Katsuo. Invariants for semifree $S^{1}$-actions, 987.

Kazdan, J. L. and Warner, F. W. Integrability conditions for $\Delta u=k-K e^{\text {aru }}$ with applications to Riemannian geometry, 819.

Kelly, Edmund. Tight equivariant immersions of symmetric spaces, 580.

Kent, G. A. A maximum principle for optimal control problems with neutral functional differential systems, 565 .

Knebusch, Manfred, Rosenberg, Alex and Ware, Roger. Structures of Witt rings, quotients of abelian group rings, and orderings of fields, 205.

Knill, R. J. On the homology of a fixed point set, 184.

Kochman, S. O. The homology of the classical groups over the Dyer-Lashof algebra, 142.

Krabbe, Gregers. An algebra of generalized functions on an open interval; two-sided operational calculus, 78.

Kwon, Y. K. and Sario, L. The $P$-singular point of the $P$-compactification for $\Delta u=P u$, 128.

Lam, P.-F. On a theorem of B. Von Kerékjarto, 230.

Lam, T. Y. See Elman, Richard. 
Lange, K., Ramsay, A. and Rota, G.-C. Frobenius reciprocity in ergodic theory, 713.

LaSalle, J. P. See Billotti, J. E.

Lashof, R. K. A nonsmoothable knot, 613.

Lawson, H. B., Jr. Codimension-one foliations of spheres, 437.

Lepowsky, J. Multiplicity formulas for certain semisimple Lie groups, 601.

Lin, S.-C. Perturbations of the unilateral shift, 621.

Lipschutz, Seymour. On conjugate powers in eighth-groups, 1050.

Loeb, P. A. A nonstandard representation of measurable spaces and $L_{\infty}, 540$.

Loos, Ottmar. Jordan triple systems, R-spaces, and bounded symmetric domains, 558.

Lundell, A. T. An exact sequence involving the Chern character, 1014.

Madsen, I. See Brumfiel, G.

Magliveras, S. S. The subgroup structure of the Higman-Sims simple group, 535.

Martin, J. C. Substitution minimal flows, 610.

Mather, J. N. On Haefliger's classifying space. I, 1107.

Meinardus, G., Reddy, A. R., Taylor, G. D. and Varga, R. S. Converse theorems and extensions in Chebyshev rational approximation to certain entire functions in $[0$, $+\infty), 460$.

Meisters, G. H. Translation-invariant linear forms and a formula for the Dirac measure, 120.

Meyers, N. G. See Adams, D. R.

Milgram, R. J. See Brumfiel, G.

Mitrović, Dragiša. The Plemelj distributional formulas, 562.

Morawetz, C. S. and Strauss, W. A. Asymptotics of a nonlinear relativistic wave equation, 797.

Moore, R. T. Generation of equicontinuous semigroups by hermitian and sectorial operators. I, 224.

- Generation of equicontinuous semigroups by hermitian and sectorial operators. II, 368.

Morris, S. A. See Diestel, Joseph.

Morrison, M. A. and Brillhart, John. The factorization of $F_{7}, 264$.

Moschovakis, Y. N. Uniformization in a playful universe, 731.

Moyer, R. D. Computation of symbols on $C^{*}$-algebras of singular integral operators, 615.

Mueller-Roemer, P. B. A note on Mackey's imprimitivity theorem, 1089.

Mycielski, Jan. See Ehrenfeucht, Andrzej.

Nakai, Mitsuru. See Glasner, Moses.

- Dirichlet finite solutions of $\Delta u=P u$, and classification of Riemann surfaces, 381. . A remark on classification of Riemann surfaces with respect to $\Delta u=P u, 527$.

Nakai, M. and Sario, L. Biharmonic classification of Riemannian manifolds, 432.

Nečas, Jindřich. On the demiregularity of weak solutions of nonlinear elliptic equations, 151.

Nitsche, J. C. C. Minimal surfaces with movable boundaries, 746.

Nussbaum, R. D. Some fixed point theorems, 360.

Nyikos, Peter. Not every 0-dimensional realcompact space is $N$-compact, 392.

Obata, Morio. The conjectures on conformal transformations of Riemannian manifolds, 265.

Ohm, Jack and Rush, D. E. The finiteness of $I$ when $R[X] / I$ is flat, 793.

Osborn, Howard. Function algebras and the de Rham theorem in PL, 386.

Overbeek, Ross. See Hughes, C. E.

Overdeck, J. M. On the nonexistence of complex Haar systems, 737.

Parthasarathy, T. Discounted and positive stochastic games, 134. 
Petrich, Mario. See Ault, Janet.

Petrie, Ted. See Browder, William.

Pickel, P. F. Finitely generated nilpotent groups with isomorphic finite quotients, 216.

Pinsky, M. A. Multiplicative operator functionals of a Markov process, 377.

Pitcher, Everett. Lefschetz formula and Morse inequalities, 1075.

Porta, Horacio. See Dubuc, E. J.

Powell, W. C. See Jech, T. J.

Pressman, I. S. Endomorphisms of exact sequences, 239.

Prolla, J. B. Weighted approximation of continuous functions, 1021.

Prosser, R. T. On the analysis and synthesis of certain abstract systems, 444 .

Putz, Robert. Boundary behavior of harmonic functions on Hermitian hyperbolic space, 473.

Quinn, Frank. $B_{\left(\text {TOP }_{n}\right)}$ - and the surgery obstruction, 596.

Ramirez, D. E. See Dunkl, C. F.

Ramsay, A. See Lange, K.

Rauch, Jeffrey. Kreiss' mixed problems with nonzero initial c'ata, 1031.

Reade, M. O. and Złotkiewicz, E. J. Koebe sets for univalent functions with two preassigned values, 103.

Rector, D. L. K-theory of a space with coefficients in a (discrete) ring, 571.

- See Gersten, S. M.

Reddy, A. R. See Meinardus, G.

Regev, Amitai. Existence of polynomial identities in $A \otimes_{F} B, 1067$.

Richardson, R. W., Jr. Deformations of Lie subgroups, 92.

Roggenkamp, K. W. Some orders of infinite lattice type, 1055.

Rosenberg, Alex. See Knebusch, Manfred.

Rosenkrantz, W. See Brezis, H.

Rota, G.-C. See Lange, K.

Rothschild, Bruce. See Golubitsky, Martin.

Rothschild, L. P. Invariant polynomials and conjugacy classes of real Cartan subalgebras, 762.

Roy, Prabir. The cardinality of first countable spaces, 1057.

Rudin, M. E. A normal space $X$ for which $X \times I$ is not normal, 246.

Ruh, E. A. Curvature and differentiable structure on spheres, 148.

Rush, D. E. See Ohm, Jack.

Sario, L. See Kwon, Y. K. . See Nakai, M.

Saxon, S. A. See Diestel, Joseph.

Schäffer, J. J. More distant than the antipodes, 606.

Scheuneman, John. Examples of compact locally affine spaces, 589.

Schoenberg, I. J. On equidistant cubic spline interpolation, 1039.

Schoenberg, I. J. and Sharma, A. The interpolatory background of the Euler-Maclaurin quadrature formula, 1034.

Schulenberger, J. R. and Wilcox, C. H. Completeness of the wave operators for scattering problems of classical physics, 777.

Schuster, Seymour. See Chartrand, Gary.

Schuur, J. D. See Chow, S.-N.

Segel, Irving. Causally oriented manifolds and groups, 958.

Shapiro, H. S. Boundary values of bounded holomorphic functions of several variables, 111.

Sharma, A. See Schoenberg, I. J.

Shiffman, Bernard, Extension of positive holomorphic line bundles, 1091. 
Simon, C. P. On a classification of a Baire set of diffeomorphisms, 783.

Singer, B. See Brezis, H.

Singletary, W. E. See Hughes, C. E.

Solazzi, R. E. Isomorphism theory of congruence groups, 164.

Stasheff, James. See Clough, Robert.

Stein, E. M. $L^{p}$ boundedness of certain convolution operators, 404.

Stein, M. R. Chevalley groups over commutative rings, 247.

Strauss, W. A. See Morawetz, C. S.

Takesaki, Masamichi. Duality and von Neumann algebras, 553.

Taylor, G. D. See Meinardus, G.

Tolimieri, Richard. Applications of the semisimple splitting, 275. - On the Selberg condition for subgroups of solvable Lie groups, 584.

Trudinger, N. S. Generalized solutions of quasilinear, differential inequalities. I. Elliptic operators, 576.

Tsuchiya, Akihiro. Characteristic classes for PL micro bundles, 531.

Unsain, Ignacio. Classification of the simple separable real L*-algebras, 462.

Varga, R. S. See Meinardus, G.

Vesterstr $\phi \mathrm{m}, \mathrm{J} \phi r \mathrm{rgen}$. Quotients of finite $W^{*}$-algebras, 235.

Wall, C. T. C. See Browder, William.

Wallach, N. R. See Greenfield, S. J.

Ware, Roger. See Knebusch, Manfred.

Warga, J. Normal control problems have no minimizing strictly original solutions, 625 .

Warner, F. W. See Kazdan, J. L.

Weinstein, Alan. Perturbation of periodic manifolds of hamilionian systems, 814 .

Welland, G. V. See Ash, J. M.

Wells, B. B., Jr. Sets of interpolation for multipliers, 220.

Wells, J. C. C $C^{1}$ partitions of unity on nonseparable Hilbert space, 804.

Wells, R. O., Jr. See Harvey, F. R.

Wente, H. C. An existence theorem for surfaces of constant mean curvature, 200.

Wilcox, C. H. See Schulenberger, J. R.

Williams, R. F. Classification of symbol spaces of finite type, 439.

Wolf, J. A. See Gromoll, Detlef.

$\mathrm{Wu}, \mathrm{H}$. A structure theorem for complete noncompact hypersurfaces of nonnegative curvature, 1070.

- See Greene, R. E.

Wulbert, Daniel. Uniqueness and differential characterization of approximations from manifolds of functions, 88 .

Yeadon, F. J. A new proof of the existence of a trace in a finite von Neumann algebra, 257.

Young, E. C. Uniqueness theorems for certain improperly posed problems, 253.

Zahler, Raphael. The Adams-Novikov spectral sequence for the spheres, 169.

Zieschang, Heiner. On extensions of fundamental groups of surfaces and related groups, 1116.

Zlotkiewicz, E. J. See Reade, M. O.

\section{BOOK REVIEWS}

Agmon, Shmuel. Review of "Maximum principles in differential equations," by M. H. Protter and H. F. Weinberger, 177.

Arbib, M. A. Review of "Recursiveness," by Samuel Eilenberg and Calvin C. Elgot, 512. 
Corwin, Lawrence. Review of "An introduction to number theory," by Harold Stark, 178.

Review of "Cohomology of groups," by Edwin Weiss, 326.

Douglas, Ronald. Review of "Equations in linear spaces," by D. Przeworska-Rolewicz and S. Rolewicz; "Theory of generalized spectral operators," by Ion Colojoara and Ciprian Foiaş; "Analyse harmonique des operateurs de l'espace de Hilbert," by Bela Sz.-Nagy and Ciprian Foiaş; "Introduction to the theory of linear nonselfadjoint operators," by I. C. Goh'berg and M. G. KreIn, 938.

Dudley, R. M. Review of "Convergence of probability measures," by P. Billingsley, 25.

Goffman, Casper. Review of "Geometric measure theory," by Herbert Federer, 27.

Schafer, R. D. Review of "Modular Lie algebras," by G. B. Seligman, 689.

Skala, Helen. Review of "Studies in geometry," by Leonard M. Blumenthal and Karl Menger, 328.

Stanley, Richard. Review of "Principles of combinatorics," by Claude Berge, 685.

Stark, H. M. Review of "Introduction to analytic number theory," by K. Chandrasekharan; "Arithmetic functions," by K. Chandrasekharan; "Multiplicative number theory," by Harold Davenport; "Sequences," by H. Halberstam and K. F. Roth, 943.

Stone, D. A. Review of "Piecewise linear topology," by J. F. P. Hudson, 521.

\section{REPORTS OF MEETINGS AND MISCELLANEOUS ARTICLES}

Antonelli, P. L., Burghelea, D. and Kahn, P. J. Errata to: "The nonfinite type of some Diffo $M^{n}, " 634$.

Bateman, P. T. Reports of Meetings of the American Mathematical Society: The November meeting in Urbana, 182; The March meeting in Chicago, 695.

Birman, J. S. Errata to: "Abelian quotients of the mapping class group of a 2-manifold," 479.

Brooks, Robin. Errata to: "The number of roots of $f(x)=a, " 479$.

Burghelea, D. See Antonelli, P. L.

Bylaws of the American Mathematical Society, 1121.

Council and Board of Trustees-1970, 175.

Curtis, Morton. Addendum to finite dimensional H-spaces, 1120.

Eagon, J. A. See Hochster, M.

Faith, Carl. Errata to: "A correspondence theorem for projective modules and the structure of simple Noetherian rings," 1120.

Floyd, E. E. and Jones, F. B. Gordon T. Whyburn 1904-1969, 57.

Gillman, Leonard. Reports of Meetings of the American Mathematical Society: The October meeting in Washington, 56.

- See Pitcher, Everett.

Gottschalk, Walter. See Pitcher, Everett.

Harrold, O. G. Reports of Meetings of the American Mathematical Society: The November meeting in Athens, 180.

Hochster, M. and Eagon, J. A. Errata to: "A class of perfect determinantal ideals," 1120.

Hodgson, J. P. E. Correction to: "Poincaré complex thickenings and concordance obstructions," 480.

Jones, F. B. See Floyd, E. E.

Kahn, P. J. See Antonelli, P. L.

Krabbe, Gregers. Correction to: "An algebra of generalized functions on an open interval; two-sided operational calculus," 479, 633.

Martin, W. T. Report of the Treasurer-1970, 710. 
Miles, Joseph. Errata to: "Representing a meromorphic function as the quotient of two entire functions of small characteristic," 634.

Pierce, R. S. Reports of Meetings of the American Mathematical Society: The November meeting in Pasadena, 181.

. See Pitcher, Everett.

Pitcher, Everett. 1970 Election Results, 176.

Pitcher, Everett and Gillman, Leonard. Reports of Meetings of the American Mathematical Society: The Annual Meeting in Atlantic City, 331.

Pitcher, Everett and Gottschalk, Walter. Reports of Meetings of the American Mathematical Society: The April meeting in New York, 697.

Pitcher, Everett and Pierce, R. S. Reports of Meetings of the American Mathematical Society: The Summer meeting in Laramie, 36; errata, 1120.

Ross, K. A. Reports of Meetings of the American Mathematical Society: The April Meeting in Monterey, 708; The June meeting in Corvallis, 709.

Statement of Ownership, Management \& Circulation, 1146.

\section{INVITED ADDRESSES}

Ax, J. B., 56.

Baker, Alan, 56. . On the class number of imaginary quadratic fields, 678.

Billingsley, P. P., 36.

Bing, R. H., 36.

Blattner, R. J., 709

Curtis, Morton. Finite dimensional H-spaces, 1.

Dugundji, James, 708.

Ellis, Robert, 695.

Fan, Ky, 709.

Gorenstein, Daniel, 697.

Gorenstein, Daniel and Harada, Koichiro. Finite simple groups of low 2-rank and the families $G_{2}(q), D_{4}^{2}(q), q$ odd, 829 .

Harada, Koichiro. See Gorenstein, Daniel.

Hemmingsen, Erik, 180.

Hofmann, K. H., 180.

Hopf, Eberhard, 331.

- Ergodic theory and the geodesic flow on surfaces of constant negative curvature, 863.

Kato, Tosio, 181.

Kato, Tosio and McLeod, J. B. The functional-differential equation $y^{\prime}(x)=a y(\lambda x)$ $+b y(x), 891$.

Kato, Tosio and McLeod, J. B. The functional-differential equation, 891.

Kesten, Harry. Some nonlinear stochastic growth models, 492.

Knopp, M. I., 695.

Kochen, Simon, 697.

Kunze, R. A., 708.

Lang, Serge. Transcendental numbers and diophantine approximations, 635.

Mahler, Kurt. A lecture on the geometry of numbers of convex bodies, 319.

Mather, John, 697.

McLeod, J. B. See Kato, Tosio.

Nachbin, Leopoldo, 331.

O'Meara, O. T., 182.

Ornstein, D. S., 181. 
Ornstein, D. S. Some new results in the Kolmogorov-Sinai theory of entropy and ergodic theory, 878 .

Petrie, T. E., 697.

Quillen, D. G., 331.

Rosenblum, Marvin and Rovnyak, James. The factorization problem for nonnegative. operator valued functions, 287.

Rovnyak, James. See Rosenblum, Marvin.

Rubel, L. A. Bounded convergence of analytic functions, 13.

Shields, A. L., 695.

Shult, E. E., 180.

Sullivan, D. P., 331.

Treves, François, 331.

Varadhan, S. S. R., 36.

Varga, R. S., 182.

Walter, J. H., 695.

Zariski, Oscar, 331.

. Some open questions in the theory of singularities, 481.

\section{Members of the Council for 1971}

Steve Armentrout, Michael Artin, Michael F. Atiyah, Hyman Bass, Paul T. Bateman, Raoul H. Bott, Felix E. Browder, Fred G. Brower, Edgar H. Brown, Jr., Alberto P. Calderón, George F. Carrier, Paul J. Cohen, Morton L. Curtis, W. H. J. Fuchs, Frederick W. Gehring, Murray Gerstenhaber, J. Wallace Givens, Jr., Irving L. Glicksberg, Oscar Goldman, Walter H. Gottschalk, Orville G. Harrold, Jr., Philip Hartman, Eugene Isaacson, Nathan Jacobson, Harry Kesten, Victor L. Klee, Jr., Joseph J. Kohn, Ray A. Kunze, Peter D. Lax, C. C. Lin, George W. Mackey, Saunders Mac Lane, W. T. Martin, Ernest A. Michael, Calvin C. Moore, Richard S. Pierce, Everett Pitcher, Murray H. Protter, Dock S. Rim, Alex Rosenberg, Kenneth A. Ross, Gian-Carlo Rota, Joseph J. Rotman, Mary Ellen Rudin, Dana S. Scott, George B. Seligman, James B. Serrin, Isadore M. Singer, Edwin H. Spanier, Elias M. Stein, Shlomo Sternberg, Michio Suzuki, P. Emery Thomas, Bertram Yood, Oscar Zariski. 